Measurement of the dynamic range of PD-L1 expression across different cores also revealed the improved sensitivity in PD-L1 detection provided by unmixing.

Conclusions The end-to-end Phenoptics staining, imaging, unmixing, and spatial analysis workflow described here provides a robust and sensitive platform for exploring the immune landscape within the tumor microenvironment.

Disclosure Information V. Goubert: None.

\section{P03.05 DEEP SPATIAL PROFILING OF THE IMMUNE LANDSCAPE OF MSI AND MSS COLORECTAL TUMORS}

SE Church, J Reeves, DR Zollinger, J McKay-Fleisch, AJ Bahrami, M Holpert*, AM White, MD Bailey, CR Merritt, M Hoang, S Warren, JM Beechem. NanString Technologies, Seattle, WA, USA

\subsection{6/jitc-2020-ITOC7.45}

Introduction In colorectal cancer (CRC) there have been many recent advances in immune related biomarkers that are both prognostic and predictive of response to immunotherapy. Microsatellite instability (MSI)/mismatch repair deficiency dMMR is present in $15-20 \%$ of CRCs and correlates with increased immunogenic mutations that often augment lymphocyte infiltration into the tumor microenvironment (TME). Additionally, location of tumor infiltrating $\mathrm{T}$ cells in two areas of the TME, the tumor center (CT) and invasive margin (IM) has also been shown to be prognostic and predictive of response to immunotherapy. Here we use multiplexed protein and RNA digital spatial profiling to elicit the immune landscape of MSI-MSS characterized CRC tumors.

Methods Forty-eight CRC tumors were analyzed for gene expression using the NanoString ${ }^{\circledR}$ nCounter ${ }^{\circledR}$ PanCancer IO $360^{\mathrm{TM}}$ Research Use Only (RUO) Gene Expression Panel and assessed for 48 cell typing and biological signatures, including MMR loss/MSI predictor and the Tumor Inflammation Signature (TIS). A subset of 18 CRC tumors (6 MSI-TIS-hi, 6 MSS-TIS-hi, 6 MSS-TIS-lo) was selected for analysis with the RUO GeoMx ${ }^{\mathrm{TM}}$ Digital Spatial Profiler (DSP) using 40 antibodies (human IO protein panel), or 84 RNA probes (human IO RNA panel). Selection of regions of interest (ROIs) in two locations, CT and IM were guided by staining with fluorescent markers (CD45, CD3, pan-CK, DNA). 300-600 $\mu \mathrm{M}$ diameter circle ROIs were selected, and in some cases segmented by pan-CK+/pan-CK-. For 2 immune hot samples contour profiling at the IM into stromal and tumor regions was performed using $1400+$ RNA probes with NGS readout.

Summary Using whole tissue gene expression analysis, we determined the TIS and IO 360 signature scores for 48 CRC tumors using PanCancer IO 360 assay. 18 tumors within this cohort were selected based on TIS status to further dissect the location-dependent immune contexture of the TME. Protein DSP confirmed loss of dMMR markers (MSH2/MLH1) and identified an increased amount of potentially suppressive macrophages (CD163+PD-L1+) in MSI-TIS-hi versus MSS-TIS-hi tumors. Segmentation of ROIs based on tumor versus stroma (pan-CK \pm ) identified samples with high proportions of tumorinvading TILs. Two MSI-TIS-hi profiled using probes against $1400+$ mRNA targets confirmed protein results (CD163 in IM) and identified tumor-related signatures corresponding to the inside of the tumor (Cytokeratins, HER2/ERBB2, MET).

Conclusions Here we show the use of novel high-plex spatial profiling to profile location and pathways in the TME of MSI and MSS CRC tumors. These findings elicit unique biology related to the location and signaling of immune cells, which have the potential to unveil targets for therapeutic combinations.

Disclosure Information S.E. Church: A. Employment (full or part-time); Significant; NanoString Technologies. J. Reeves: A. Employment (full or part-time); Significant; NanoString Technologies. D.R. Zollinger: A. Employment (full or parttime); Significant; NanoString Technologies. J. McKayFleisch: A. Employment (full or part-time); Significant; NanoString Technologies. A.J. Bahrami: A. Employment (full or part-time); Significant; NanoString Technologies. M. Holpert: A. Employment (full or part-time); Significant; NanoString Technologies. A.M. White: A. Employment (full or parttime); Significant; NanoString Technologies. M.D. Bailey: A. Employment (full or part-time); Significant; NanoString Technologies. C.R. Merritt: A. Employment (full or part-time); Significant; NanoString Technologies. M. Hoang: A. Employment (full or part-time); Significant; NanoString Technologies. S. Warren: A. Employment (full or part-time); Significant; NanoString Technologies. J.M. Beechem: A. Employment (full or part-time); Significant; NanoString Technologies.

\section{P03.06 PATTERN OF KI67 ${ }^{+}$EXPANDING CD8 ${ }^{+}$CYTOTOXIC T CELLS IN HEALTHY TISSUES, INFLAMMATION AND THE CANCER MICROENVIRONMENT}

K Möller, M Lennartz, R Abu-Hashem, NC Blessin, T Mandelkow, E Bady, C Hube-Magg*, R Simon, G Sauter, C Fraune, TS Clauditz, F Büscheck, AM Luebke. University Medical Center Hamburg-Eppendorf, Hamburg, Germany

\subsection{6/jitc-2020-ITOC7.46}

Background Expansion of $\mathrm{CD}^{+}$cytotoxic $\mathrm{T}$ lymphocytes is a prerequisite for anti-cancer immune activity. In the era of immune checkpoint therapy, profound knowledge of the dynamics of $\mathrm{CD}^{+}$has regained considerable interest. However, systematically acquired data on $\mathrm{CD}^{+}$proliferation in large sets of normal and diseased tissues are sparse.

Materials and Methods Here, we applied multiplex fluorescence immunohistochemistry to conventional large sections and tissue microarrays in order to quantitate $\mathrm{Ki} 67^{+} \mathrm{CD} 8^{+}$cells in $>20$ different compartments of normal lymphoid tissues, 7 types of inflammatory diseases and 785 cancers.

Results In most normal lymphoid tissues (tonsil, lymph node, thymus, Peyer's patches, spleen, colon, appendix) the percentage of $\mathrm{Ki} 67^{+} \mathrm{CD} 8^{+}$cells typically did not exceed $3 \%$. The percentage of $\mathrm{Ki} 67^{+} \mathrm{CD} 8{ }^{+}$cells was markedly higher $(45 \%)$ in the immune-active cortex of the thymus, however. In inflammatory conditions (including Hashimoto thyroiditis, Lichen sclerosus of the penis, sarcoidosis, sialadenitis, IgG4 pancreatitis, Crohn's disease and eczema), the percentage of $\mathrm{Ki}^{+} 7^{+} \mathrm{CD}^{+}$cells was much more variable and often sharply higher than in normal tissues. It ranged from $0.5 \%$ in one patient with sialadenitis to $19 \%$ in the intraepithelial compartment of Crohn's disease. In 765 colorectal cancers, the fraction of $\mathrm{Ki} 67$ positive $\mathrm{CD} 8^{+}$cytotoxic $\mathrm{T}$ cells ranged from 0 to $100 \%$ (mean: $20.6 \%$ ). A high fraction of $\mathrm{Ki}^{2} 7^{+} \mathrm{CD}^{+}$cells was significantly associated with microsatellite instability $(\mathrm{p}<0.0001)$, low pT stage $(\mathrm{p}<0.0001)$ and absence of nodal metastases $(p=0.0005)$.

Conclusions In summary, our data show a variable increase of the fraction of proliferating $\mathrm{CD}^{+} \mathrm{T}$ cells in cancers and in 
inflammatory diseases as compared to healthy secondary lymphoid organs. The striking link with microsatellite instability and unfavorable tumor features suggest a potential clinical utility of assessing $\mathrm{Ki} 67^{+} \mathrm{CD} 8^{+}$in colorectal cancer.

Disclosure Information K. Möller: None. M. Lennartz: None. R. Abu-Hashem: None. N.C. Blessin: None. T. Mandelkow: None. E. Bady: None. C. Hube-Magg: None. R. Simon: None. G. Sauter: None. C. Fraune: None. T.S. Clauditz: None. F. Büscheck: None. A.M. Luebke: None.

\section{P03.07 FAST AUTOMATED MICROFLUIDIC-BASED MULTIPLEXED IMMUNOFLUORESCENCE FOR TUMOR MICROENVIRONMENT ANALYSIS}

${ }^{1}$ A Kehren*, ${ }^{2}$ MG Procopio, ${ }^{1} B$ Pelz, ${ }^{1} Z$ Siddiqui, ${ }^{3} \mathrm{~K}$ Roman, ${ }^{1} \mathrm{~S}$ Adnane, ${ }^{1} \mathrm{~S}$ Brajkovic, ${ }^{3} \mathrm{C}$ Hoyt, ${ }^{1}$ DG Dupouy, ${ }^{2} \mathrm{~A}$ Soltermann. ' Lunaphore Technologies SA, Lausanne, Switzerland; ${ }^{2}$ Institut für Klinische Pathologie Universitätsspital Zürich, Zürich, Switzerland; ${ }^{3} A k o y a$ Biosciences, Hopkinton, MA, USA

\subsection{6/jitc-2020-ITOC7.47}

Background Immuno-oncology and targeted molecular therapies have acquired a central role in the treatment of multiple cancers. Consequently, high-throughput biomarker analysis and tumor immune profiling have seen an increased demand. Multiplexed immuno-assays are a powerful tool to address these needs, but still time- and resource-consuming. Our goal is to develop a fast and automated high-plex fluorescent immunostaining procedure, using a microfluidic-based device, that can be easily implemented as routine assay.

Materials and Methods Protocol optimization has been performed on FFPE sections of human tonsil. Slides were manually deparaffinized before being entirely processed (antigenretrieval, staining, elution and counterstaining) by Lunaphore's autostainer, LabSat ${ }^{\mathrm{TM}}$. The $\mathrm{OPAL}^{\circledR}$ tyramide signal amplification (TSA) system was used as detection method. Signal analysis was done on Mantra ${ }^{\circledR}$ workstation. The 6-plex panel was composed of FoxP3, PD-L1, PD-1, CD68, CD8 and pan-CK, plus DAPI counterstaining. Protocols were subsequently transferred on NSCLC representative specimens and finally assessed on a TMA cohort.

Results Our platform allowed to reduce drastically the incubation times due to active transport of reagents across the tissue. Thereby, the automated 6-plex assay could be performed in less than $4 \mathrm{~h} 30 \mathrm{~min}$, within the timeframe of a single IHC standard assay. Protocol optimization resulted in high signalto-background ratio for each marker and removal of previous step antibodies over 99\%. LabSat ${ }^{\mathrm{TM}}$ also guaranteed remarkable signal uniformity, even over large tissue sections with less than $10 \%$ signal gradient over $1 \mathrm{~cm}$. On NSCLC samples, the detected pattern and expression level for all six biomarkers were comparable to the standard chromogenic stainings performed with standard automated tissue stainer.

Conclusions LabSat ${ }^{\mathrm{TM}}$ autostainer enables multistaining runs in a timely manner, opening the perspective of rapid simultaneous detection of multiple markers in their morphological context on a routine-based approach. This versatile analysis tool can offer a better and more quantitative understanding of tumor heterogeneity and microenvironmental interactions, allowing advances in targeted therapy for lung cancer as well as broader spectrum of malignancies.

Disclosure Information A. Kehren: A. Employment (full or part-time); Significant; Lunaphore Technologies SA. C. Other Research Support (supplies, equipment, receipt of drugs or other in-kind support); Significant; Akoya Biosciences. M.G. Procopio: B. Research Grant (principal investigator, collaborator or consultant and pending grants as well as grants already received); Significant; KTI grant (Schweiz). B. Pelz: A. Employment (full or part-time); Significant; Lunaphore Technologies SA. C. Other Research Support (supplies, equipment, receipt of drugs or other in-kind support); Significant; Akoya Biosciences. Z. Siddiqui: A. Employment (full or part-time); Modest; Lunaphore Technologies SA. K. Roman: A. Employment (full or part-time); Significant; Akoya Biosciences. S. Adnane: A. Employment (full or part-time); Modest; Lunaphore Technologies SA. S. Brajkovic: A. Employment (full or part-time); Significant; Lunaphore Technologies SA. C. Hoyt: A. Employment (full or part-time); Significant; Akoya Biosciences. D.G. Dupouy: A. Employment (full or part-time); Significant; Lunaphore Technologies SA. E. Ownership Interest (stock, stock options, patent or other intellectual property); Significant; Lunaphore Technologies SA. A. Soltermann: A. Employment (full or part-time); Significant; Institut für Klinische Pathologie Universitätsspital Zürich.

\section{P03.08 OMENTAL FAT IN OVARIAN CANCER POTENTIALLY INDUCES LYMPHANGIOGENESIS}

${ }^{1}$ BMA Lenoir*, ${ }^{2}$ V Starrach, ${ }^{2} \mathrm{D}$ Ferber, ${ }^{1} \mathrm{M}$ Suarez-Carmona, ${ }^{3} \mathrm{~S}$ Schott, ${ }^{1} \mathrm{I}$ Zörnig, ${ }^{1,2} \mathrm{D}$ Jäger, ${ }^{1,2} \mathrm{~N}$ Halama. ${ }^{1} D K F Z$, Heidelberg, Germany; ${ }^{2} N C T$, Heidelberg, Germany; ${ }^{3}$ University hospital, Heidelberg, Germany

\subsection{6/jitc-2020-ITOC7.48}

Background Ovarian cancer metastasis occurs by direct multifocal seeding in the peritoneum as well as by migration through the lymphatic system. High grade ovarian carcinoma patients present with distant metastases. Significant risk factors for the development of those are stage, grade, and lymph node involvement. An increase of the number of lymphatic vessels is shown in ovarian tumors and these vessels seem implicated in tumor progression. While the tropism of ovarian cancer cells for fat is well described, the potential impact of a fatty microenvironment on the dissemination of tumor cells via lymphatic vessels has, to our knowledge, never been investigated yet. In this study, we examined the effect of omental fat on lymphangiogenesis in ovarian carcinoma.

Materials and Methods To examine the effect of omental fat on lymphangiogenesis in OC we used a cohort of 80 human specimens. We analysed lymphatic vessels histologically with D2-40 and Lyve-1 markers. We also developed a healthy fat tissue explant culture model and treated explants with ascites of patient with OC before analysis. We analysed by fluorescence stainings the co expression of adipose derived stem cells (ASCs) and lymphatic markers in these explants.

Results We observed a higher density of tumor-associated vessels, especially lymphatic vessels in OC in contact with the omentum; mainly localized along the adipose tissue. We also measured a higher secretion of VEGF-C in tissues with fat compared to tissues without fat. Healthy fat tissues treated with ascites show an increase of number of ASCs, some of them express lymphatic markers such as D2-40 and Lyve-1. In a clinical trial of patients with OC treated by Bevacizumab, we observed a decrease of the number of lymphatic vessels in correlation with a decrease of the inflammation around the fat tissue.

Discussion We saw an increase in the number of lymphatic vessels in ovarian carcinoma infiltrating fat. These vessels are 